\title{
A predictive model for the sensory aroma characteristics of flue-cured tobacco based on a back-propagation neural network
}

\author{
Conglian $\mathrm{He}^{1,2} \cdot$ Ruoxing Chen ${ }^{3} \cdot \mathrm{Ke} \mathrm{Ren}^{1,2} \cdot \mathrm{Gaokun}_{\mathrm{Zhao}^{1}} \cdot$ Chenggang $\mathrm{He}^{2} \cdot$ Binbin $\mathrm{Hu}^{1}$. Congming Zou ${ }^{1}$. \\ Yonglei Jiang ${ }^{1} \cdot$ Yi Chen $^{1}$
}

Received: 21 April 2020 / Accepted: 8 October 2020 / Published online: 23 October 2020

(c) Springer Nature Switzerland AG 2020

\begin{abstract}
In this study, aroma quality, aroma quantity, diffusiveness and offensive odor predictions were modeled using a back propagation neural network (BPNN). The model was built from chemical components and sensory quality data features of flue-cured tobacco leaf samples. The results showed that the BPNN model adapted the optimized hidden layer well and had excellent generalization ability and robustness. The coefficients of determination $\left(R^{2}\right)$ for the indices of tobacco aroma characteristics based on the BPNN model, namely, aroma quality, aroma quantity, diffusiveness and offensive odor, were all above 0.7. The accuracies of the model parameters (mean squared error, regression mean standard error , and mean absolute error) were better than those of the model built using stepwise regression analysis. These results indicate that the BPNN prediction model is reliable and can accurately predict the sensory aroma characteristic qualities of flue-cured tobacco leaf.
\end{abstract}

\section{Graphic abstract}

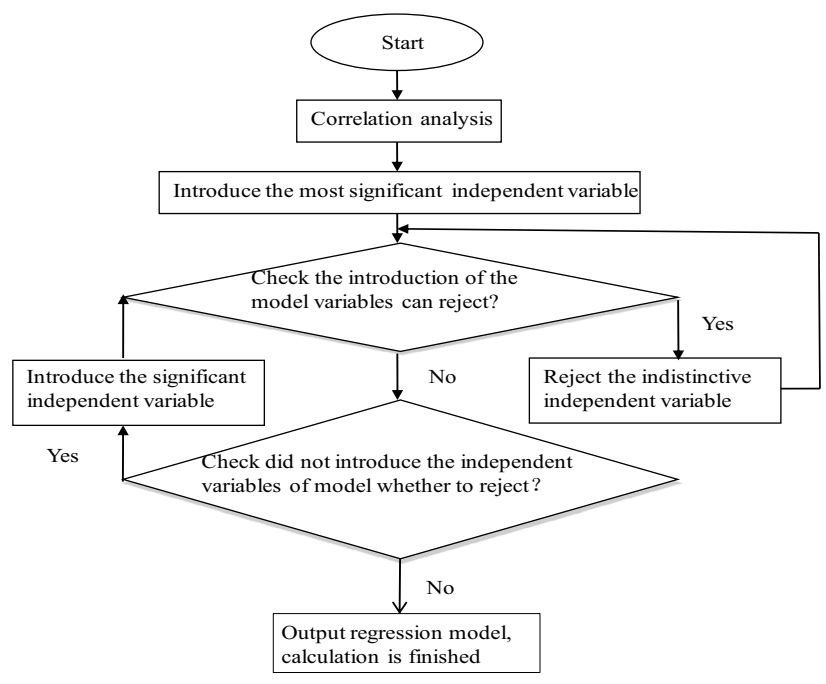

Conglian He and Ruoxing Chen have contributed equally to this manuscript.

Yi Chen, cytobacco007@sina.com | ${ }^{1}$ Yunnan Academy of Tobacco Agricultural Sciences, Agronomic Research Center, Yuan Tong Jie No. 33, Kunming 650021, Yunnan, China. ${ }^{2}$ Yunnan Agricultural University, Kunming 650201, Yunnan, China. ${ }^{3}$ Research center of Jiangxi tobacco Industrial Co., Ltd of China, Nanchang 330096, Jiangxi, China. 
Keywords Back-propagation neural network · Flue-cured tobacco · Chemical components · Sensory quality · Stepwise regression analysis

\section{Introduction}

Flue-cured tobacco is the most important type of tobacco and accounts for $66 \%$ of all types of tobacco produced globally [28]. The evaluation of FCTL quality is largely based on sensory evaluations of quality and style. The quality characteristics include aroma, smoke type (smoothness degree, softness degree, sense of mellowness) and taste (stimulation, dry sensation, remaining taste). The aroma characteristic sensory evaluation indicator comprises aroma quality, aroma quantity, diffusiveness and offensive odor. The chemical components of FCTL, such as total sugar, reducing sugar, total nitrogen, total nicotine, chlorine, and potassium, have a large influence on the FCTL quality $[26,27]$. Therefore, it is great important to improve the correlation between chemical components and sensory quality indices for the rational utilization of tobacco leaves.

The judgment of sensory quality is mainly based on expert assessments. However, smoking is harmful to the expert's health. Moreover, through expert assessments alone, it is difficult to establish the relationship between the chemical components and sensory characteristics of flue-cured tobacco leaves. The study of the relationship between the chemical components of flue-cured tobacco leaves and the quality of evaluation analyses is a key field with a long history. However, some imperfections have been reported from previously proposed techniques such as SAR [23], principal component analysis [24], canonical correlation analysis [7], gray correlation analysis [32], fuzzy mathematics [35] and so on. These methods are helpful in qualitative analysis but cannot build a reliable and accurate model of the complex relationship between chemical components and sensory quality indices.

As mathematical statistical techniques have developed, artificial neural networks (ANNs) have been widely applied in many fields, particularly a model called the back-propagation neural networks (BPNN). BPNNs are commonly used in food production, processing and for food quality assessments. Traditional data processing approaches typically fail in these applications and have relatively high costs. Due to its inherent capabilities, a BPNN can be successfully used to predict properties in a wide range of fields, such as chemistry $[2,26,27]$, economics [6], medicine [15], engineering [8], psychology [25], and food quality assessment [1]. For instance, five different tea samples have been classified using a BPNN, achieving an accuracy rate of $88 \%$ [5]. Another study classified several wine samples, and different quality parameters have been predicted with a BPNN based on gas sensor measurements [29]. These studies show that BPNN model has wide availability and relatively high accuracy.

In this study, BPNN and SRA models of the chemical indices for flue-cured tobacco leaves for SAC quality were established from the same specimen data. The data in this study were acquired from eight different regions covering the major tobacco production regions in China. By comparing and screening the best evaluation model, the goal of this study was to provide both theoretical and practical bases by which industrial enterprises can improve the quality of flue-cured tobacco leaves across various raw material bases and make more efficient use of the leaves.

\section{Materials}

\subsection{Sample materials and analysis}

Flue-cured tobacco leaves variety K326 were collected from eight areas belonging to Yunnan China Tobacco Co., Ltd. in 2018; Zunyi Guizhou (latitude $27^{\circ} 46^{\prime} \mathrm{N}$, longitude $113^{\circ} 15^{\prime} \mathrm{E}, 1200 \mathrm{~m}$ above sea level), Chenzhou Hunan (latitude $25^{\circ} 50^{\prime} \mathrm{N}$, longitude $106^{\circ} 55^{\prime} \mathrm{E}, 1100 \mathrm{~m}$ above sea level), Pingdingshan Henan (latitude $33^{\circ} 78^{\prime} \mathrm{N}$, longitude $113^{\circ} 33^{\prime} \mathrm{E}, 248 \mathrm{~m}$ above sea level), Sanming Fujian (latitude $26^{\circ} 13^{\prime} \mathrm{N}$, longitude $117^{\circ} 39^{\prime} \mathrm{E}, 800 \mathrm{~m}$ above sea level), Liangshan Sichuan (latitude $27^{\circ} 52^{\prime} \mathrm{N}$, longitude $102^{\circ} 03^{\prime}$ $\mathrm{E}, 1100 \mathrm{~m}$ above sea level) and Baoshan Yunnan (latitude $24^{\circ} 51^{\prime} \mathrm{N}$, longitude $99^{\circ} \mathrm{E}, 1673 \mathrm{~m}$ above sea level) (Fig. 1). Each of the 216 samples weighed $3 \mathrm{~kg}$. Half of each sample $(1.5 \mathrm{~kg})$ was shredded, screened with a 60 -mesh sieve, and analyzed by a chemical component test to obtain total sugar, reducing sugar, total nitrogen, total nicotine, chlorine, and potassium measurements [10]. All the chemical indicator analysis methods complied with current industrial standards. The remaining portion of the tobacco samples was used to make single-material cigarettes for sensory quality evaluations. The sensory aroma characteristics were evaluated by 7 experts from Yunnan China Tobacco Co. Ltd., based on the sensory evaluation methods for the quality style characteristics of flue-cured tobacco leaves. The $0-5$ equidistance scale scoring method was adopted to make a quantitative evaluation of the aroma characteristics (Table 1). Among these, there were 9 evaluation indices for offensive odor, and most of the samples were given a score of 3 for their offensive odor. After adding the scores of all the offensive odor indices, standardization processing was conducted. 
Fig. 1 Sampling location of this study. Flue-cured tobacco leaves samples were collected from 6 province, Chenzhou city in Hunan province, Xuchang city in Henan province, Sanming city in Fujian province, Zunyi city in Guizhou province, Liangshan city in Sichuan province, Baoshan city in Yunnan province, China
Table 1 Evaluation index and scoring scale for src of FCTL

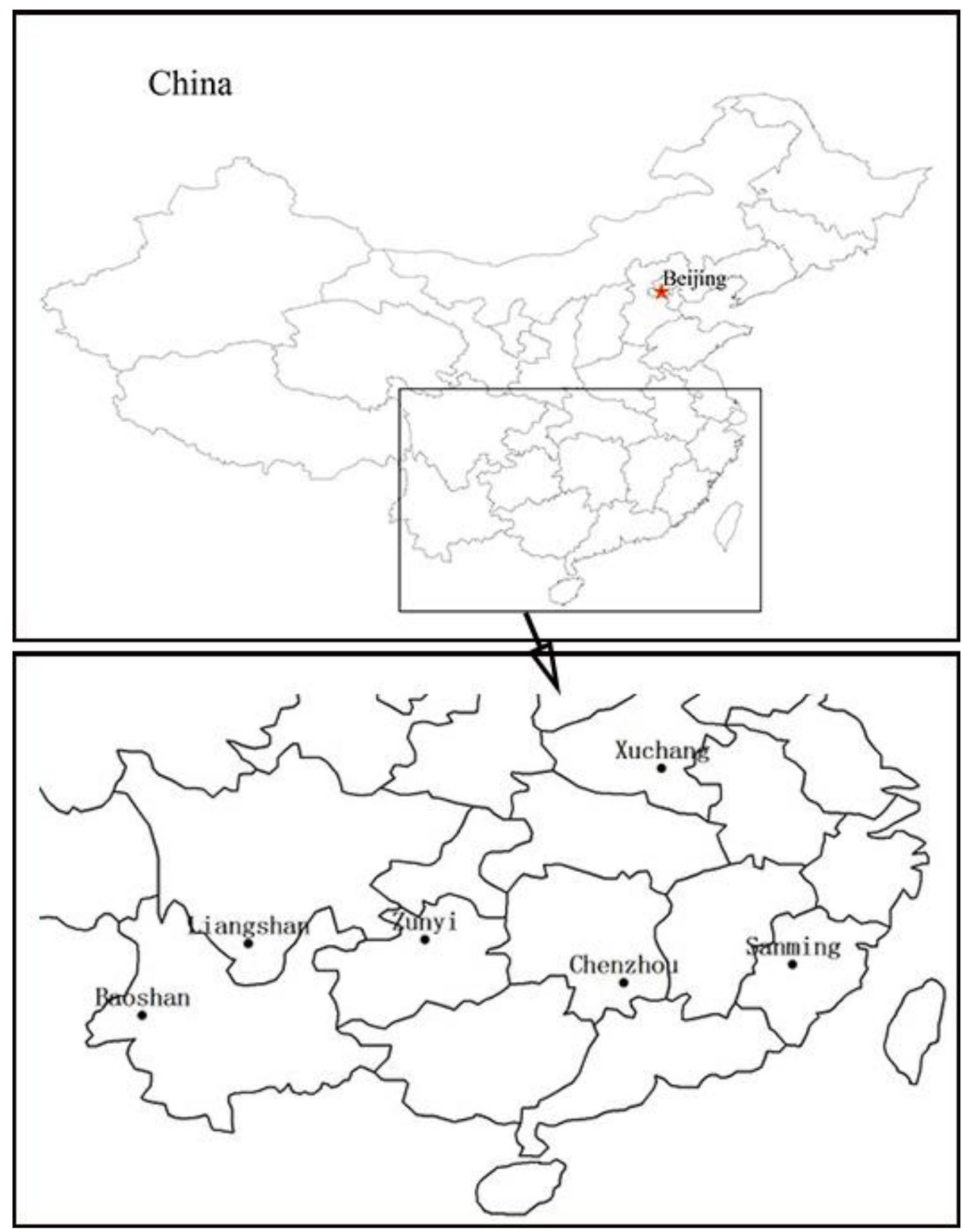

\begin{tabular}{|c|c|c|c|}
\hline \multirow[t]{2}{*}{ Evaluation indicator } & \multicolumn{3}{|l|}{ Scale value } \\
\hline & $0-1$ & $2-3$ & $4-5$ \\
\hline Aroma quantity & Little $\sim$ slight & Slight $\sim$ yet sufficient & Yet sufficient $\sim$ abundant \\
\hline Aroma quality & Inferior poor & Slightly better $\sim$ good & Quite good better \\
\hline Diffusiveness & $\begin{array}{l}\text { Oppressive } \sim \text { more } \\
\text { oppressive }\end{array}$ & Slight $\sim$ diffusiveness & Quite diffusiveness $\sim$ fine \\
\hline Offensive odor & None $\sim$ slight & Slight $\sim$ perceptible & Quite heavy heavy \\
\hline
\end{tabular}




\section{Methods}

\subsection{BPNN model building}

According to the Chinese national standard for flue-cured tobacco leaves (GB2635-1992), 10 routine chemical indices of tobacco were selected, including total sugar, reducing sugar, nicotine, total nitrogen, chlorine, potassium, ratio of total sugar to reducing sugar, ratio of sugar to nicotine, ratio of nitrogen to nicotine, and ratio of potassium to chlorine as the input features for the models. Moreover, the SAC indices of flue-cured tobacco leaves, namely, aroma quantity, aroma quality, diffusiveness and offensive odor, were selected as the output. The correlations between the routine chemical indices and the SAC index of FCTL are listed in Table 2.
Table 2 Correlation relationship between routine chemical indices and SAC indices of FCTL

\begin{tabular}{lcccc}
\hline Chemical indicator & Aroma quantity & Aroma quality & Diffusiveness & Offensive odor \\
\hline Total sugar & $0.59^{* *}$ & $0.45^{* *}$ & $0.45^{* *}$ & $-0.25^{* *}$ \\
Reducing sugar & $0.53^{* *}$ & $0.45^{* *}$ & $0.40^{* *}$ & $-0.20^{* *}$ \\
Nicotine & $0.18^{* *}$ & $0.62^{* *}$ & $0.38^{* *}$ & $0.19^{* *}$ \\
Total nitrogen & -0.08 & 0.05 & 0.06 & -0.02 \\
Potassium & $-0.28^{* *}$ & $-0.49^{* *}$ & $-0.37^{* *}$ & 0.06 \\
Chlorine & $-0.17^{*}$ & -0.05 & -0.05 & 0.06 \\
Ratio of total sugar to reducing sugar & $-0.14^{*}$ & 0.00 & -0.12 & 0.09 \\
Ratio of sugar to nicotine & $0.23^{* *}$ & $-0.24^{* *}$ & -0.07 & $-0.34^{* *}$ \\
Ratio of nitrogen to nicotine & $-0.24^{* *}$ & $-0.49^{* *}$ & $-0.33^{* *}$ & $-0.16^{*}$ \\
Ratio of potassium to chlorine & -0.05 & $-0.21^{* *}$ & $-0.21^{* *}$ & 0.01 \\
\hline
\end{tabular}

"**and"**" is the significant difference of the T test on the level of $P<0.05$ and $P<0.01$ respectively

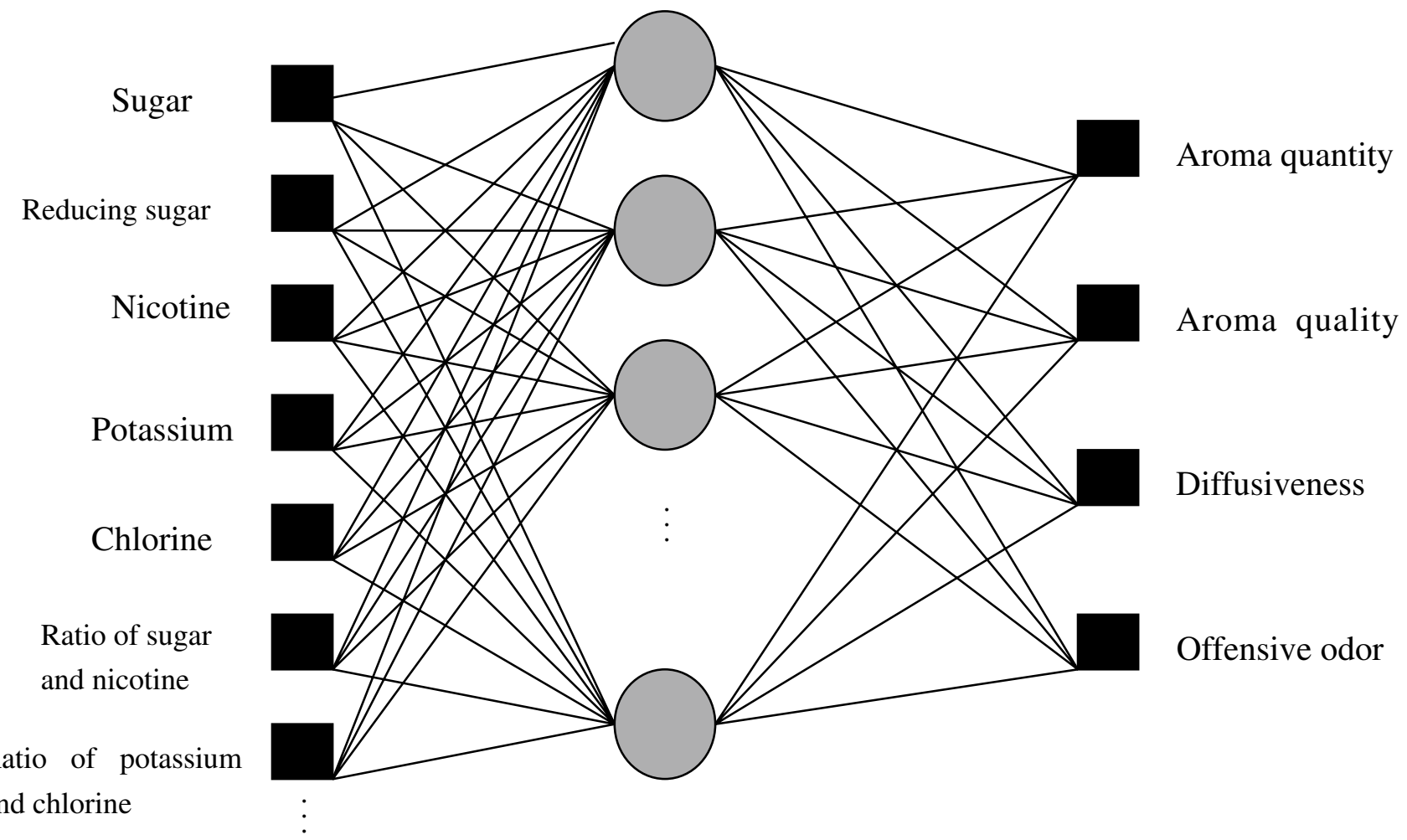

Input layer Hidden layer Output layer

Fig. 2 Topological structure of back-propagation neural network 
As shown in Table 2, the SAC indices are closely related to the routine chemical indices of FCTL, with high reliability $(P<0.01)$. Then, the previously mentioned chemical indices were used as inputs for the BPNN model. According to the Kolmogorov theorem, a BPNN with a single hidden layer structure (Fig. 2) was adopted in this study to build a model to predict aroma quantity, aroma quality, diffusiveness and offensive odor. The complete model structure includes three layers: an input layer, a hidden layer and an output layer. The aroma quality model had a training topological structure of $8 \times S \times 1$; the number of hidden nodes was subject to the experiment $[9,22,33]$.

To address the inconsistent units and orders of magnitudes between the input variables of the neural network, a normalization method was adopted to make the larger inputs fall within the range of the larger neuron transfer function gradient. Namely, the input $X$ and the output $Y$ were respectively normalized as follows:

$x_{i j}=\frac{\bar{X}_{i j}-x_{\min }}{X_{\max }-X_{\min }}$

where $X_{i j}$ is the data after the input changes, ranging from 0 to $1, \bar{X}_{i j}$ is the input test data, $X_{\max }$ is the maximum value of the $X$ characteristic of all specimens, and $X_{\min }$ is the minimum value of the $X$ characteristic of all specimens. Similarly,

$Y_{i j}=\frac{\bar{Y}_{i j}-Y_{\min }}{Y_{\max }-Y_{\min }}$

where $Y_{i j}$ is the data after the output changes, ranging from 0 to $1, \bar{Y}_{i j}$ is the output test data, $Y_{\max }$ is the maximum value of all output specimens, and $Y_{\min }$ is the minimum value of all output specimens.

The BPNN model outputs its results by forward propagation. The results are used for back-propagation, in which the weight and threshold connections between the neurons in each layer are updated when the results of the output layer do not meet the expected results. The model iterates over the entire process until the signal error reaches an acceptable range. The weights are revised as follows:

$E_{i j}=\left(Y_{i j}^{\prime}-Y_{i j}\right)^{2}$

$\left.E_{i}=\sum_{j=1}^{M}\left(Y_{i j}^{\prime}-Y_{i j}\right)^{2}\right)$

$E_{j}=\frac{1}{N v} \sum_{i=1}^{N v} \sum_{j=1}^{M}\left(Y_{i j}^{\prime}-Y_{i j}\right)^{2}=\frac{1}{N v} \sum_{i=1}^{N v} E_{i j}$
$w(j+1)=w(j)+\Delta w(j+1)$

$\Delta w(j+1)=a \frac{\partial E}{\partial w}+\eta \Delta w(j)$

where $N v$ is the training sample array, $i$ is the i set of samples, $Y_{i j}^{\prime}$ is the desired output of output node $j$ for sample $\mathrm{i}, Y_{i j}$ is the actual output of output node $\mathrm{j}$ for sample $\mathrm{j}, E_{i}$ is the mean square error of the $i$ set, $E_{j}$ is the mean square error of the $\mathrm{j}$ set of the output node for all samples, $E$ is the total error in all samples, $a$ is the learning rate, and $\eta$ is a momentum factor.

The activation function from the input layer to the hidden layer is a nonlinear sigmoid function $\left(f(x)=\frac{1}{1+\mathrm{e}^{-x}}\right)$, and the activation function from the hidden layer to the output layer is the purelin function. Because BPNNs are prone to fall into local minima and have slow convergence, the momentum method and a self-adapting learning-rate adjustment strategy were adopted: the learning rate selected for the model was 0.001 , the learning rate increment was 10 for, the learning rate decrement was 0.1 , the maximum iterations were set to $5000 \mathrm{for}$, and the target tolerance was set to 0.001 . The BPNN model was coded in Matlab $8.0[4,12]$.

\subsection{SRA model building}

To provide a comparison for analyzing the BPNN model accuracy, a mathematical statistical method was adopted to establish a multiple regression model of the sensory aroma characteristics for the main chemical components of FCTL using the following regression equation as follows:

$y=\beta_{0}+\beta_{1 \times 1}+\beta_{2 \times 2}+\cdots+\beta m \times m+\varepsilon$

where $\beta_{0}, \beta_{1}, \ldots, \beta_{m}$ is the regression coefficient, $\epsilon$ is a random variable (the remaining parameters), and $x_{1}, x_{2}, \ldots, x_{m}$ is the set of explanatory variables for the SRA model.

The stepwise regression method was used for the model built by (8), and the explanatory variable set $x_{m}$ was significantly affected $y$. Variables can be added to or deleted from the regression model based on their significance, and this approach was used to confirm the explanatory variable set for the regression model. The steps of the stepwise regression algorithms were achieved as follows:

1. The routine chemical indices of FCTL $\beta_{0}$ were used as initial values to start the regression analysis.

2. Variables were added into the regression model when they passed an $\mathrm{F}$ test, which indicates that an explanatory variable $x_{i}$ (not yet present in the model) affects y significantly. Only one explanatory variable is intro- 
duced in each step; in this manner, a partial regression equation can be established.

3. When an explanatory variable was introduced, the partial regression sum of the squares of the previously introduced variables in this equation are tested individually. The newly introduced variable is deleted from the equation if the result of test $T$ showed that the effect of the added variable was insignificant. New variables that significantly affected $y$ are reconsidered for the equation when all the arguments in the partial regression equation had significant effects on $y$ and were saved for the next round.

4. By repeating steps 2 and 3 consistently, the stepwise regression process continues until no variable can deleted and no additional explanatory variables can be introduced. The SRA model for sensory aroma characteristics based on the main chemical components of FCTL is shown in Fig. 3 [30].

\subsection{Performance analysis}

To objectively evaluate the models' performances, 5 metrics were selected for inspection, including the sum of

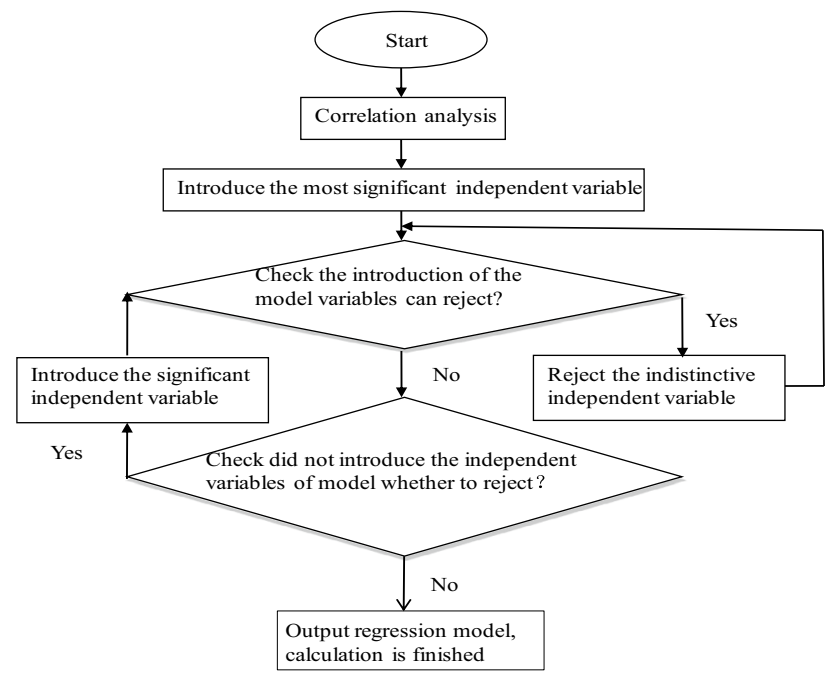

Fig. 3 The flow chat of stepwise regression analysis squared errors (SSE), mean squared error (MSE), regression mean standard error (RMSE), mean absolute error (MAE) and the coefficient of determination $\left(R^{2}\right)[16,17]$, which are calculated as follows:

SSE $=\sum_{i=1}^{n}\left(Y_{\text {estimated }}-Y_{\text {targ et }}\right)^{2}$

MSE $=\frac{1}{n} \sum_{i=1}^{n}\left(Y_{\text {estimated }}-Y_{\text {targ et }}\right)^{2}$

$R M S E=\sqrt{\frac{\sum_{i=1}^{n}\left(Y_{\text {estimated }}-Y_{t \text { arget }}\right)^{2}}{n}}$

$R^{2}=1-\left(\frac{\sum_{i=1}^{n}\left(Y_{\text {estimated }}-Y_{t \text { arget }}\right)^{2}}{\sum_{i=1}^{n}\left(Y_{\text {target }}\right)^{2}}\right)$

$M A E=\frac{\sum_{i=1}^{n}\left|Y_{\text {estimated }}-Y_{t \text { arg et }}\right|}{n}$

where $Y_{\text {target }}$ represents the true values of sensory aroma characteristics used to train the model, and $Y_{\text {estimated }}$ represents the predicted values of sensory aroma characteristics output by the model.

\section{Results}

\subsection{Selecting the number of neurons in the hidden layer of the BPNN}

The number of neurons in the input layer and output layer of BPNN is dependent on the dimensionality of the input and target specimens [19]. Of the aforementioned data specimens, $70 \%$ were adopted as a training set, and $30 \%$ were adopted as the testing set [20]. Table 3 shows the number of hidden layers when the SSE of the simulated value and the measured value of the specimens for aroma quality, aroma quantity, diffusiveness and offensive odor

Table 3 SSE of test specimen simulation on SAC indices of different neuron numbers of hidden layers

\begin{tabular}{lcccccrr}
\hline Indicator & $\begin{array}{l}\text { Number of hid- } \\
\text { den layer 6 }\end{array}$ & $\begin{array}{l}\text { Number of hid- } \\
\text { den layer 7 }\end{array}$ & $\begin{array}{l}\text { Number of hid- } \\
\text { den layer 8 }\end{array}$ & $\begin{array}{l}\text { Number of hid- } \\
\text { den layer } 9\end{array}$ & $\begin{array}{l}\text { Number of hid- } \\
\text { den layer 10 }\end{array}$ & $\begin{array}{l}\text { Number of hid- } \\
\text { den layer } 11\end{array}$ & $\begin{array}{l}\text { Number of } \\
\text { hidden layer } \\
12\end{array}$ \\
\hline Aroma quality & 28.00 & 37.91 & 87.00 & 45.70 & 155.49 & 86.65 & 134.16 \\
Aroma quantity & 35.03 & 51.42 & 568.31 & 35.59 & 104.92 & 84.93 & 222.15 \\
Diffusiveness & 56.97 & 310.80 & 40.50 & 90.83 & 86.65 & 92.92 & 57.26 \\
Offensive odor & 139.42 & 226.32 & 190.93 & 123.26 & 144.27 & 146.23 & 276.34 \\
\hline
\end{tabular}


was minimized. When the number of aroma quality neurons was 6 , the corresponding SSE was 28.00 , which is the minimum value. Therefore, 6 was selected as the number of neurons in the hidden layer, leading to a network structure of 8-6-1. Similarly, the network structure for aroma quantity was 7-6-1, whereas the network structure for diffusiveness was 6-8-1, and the network structure for offensive odor was 5-9-1.

\subsection{Simulation forecast of the hidden layer of BPNN}

After optimizing the structure of the BPNN, the modeling data specimens were adapted to train and inspect the network. Among the 216 samples, 150 were selected for network training, and the remaining 66 samples were selected for network testing. The results are shown in Fig. 4. After the neural network training, the simulated and measured values of aroma quality, aroma quantity, diffusiveness and offensive odor coincided with each other. Thus, this method for predicting the sensory aroma characteristic index of FCTL via BPNN was feasible.

\subsection{Simulation result of the SRA model}

According to the analysis of the correlation of routine chemical indices and SAC indices of FCTL in Table 2. The 9 chemical indices, including total sugar, reducing sugar, nicotine and ratio of nitrogen to nicotine, chlorine, potassium, ratio of total sugar to reducing sugar, ratio of sugar to nicotine, and ratio of potassium to chlorine having significant correlation with the SAC indices. Therefore, the 9 chemical indices were selected as the independent variables and the SAC indices were adopted as the dependent variables. Table 4 showed the optimal linear regression equations, all of which were established by constructing the SRA using the routine chemical indices and SAC indices of FCTL. According to the results of and F test, there were highly significant differences between the multiple linear regression equations. Because the regression equation removes the influence of other factors, it has good reliability for judging the nature and closeness of the relationships between variables.

\subsection{Comparison of the results of both models}

To further verify the accuracy of the BPNN and SRA models a comparative analysis of the simulated results of the two models was conducted using all specimens. According to Table 5: (1) the predication accuracy of the SRA model was obviously inferior to that of the BPNN model, indicating that the model specimen set is a non-linear system; (2) in the non-linear model based on the BPNN, the $\mathrm{R}^{2}$ of all the indices for the tobacco aroma characteristics
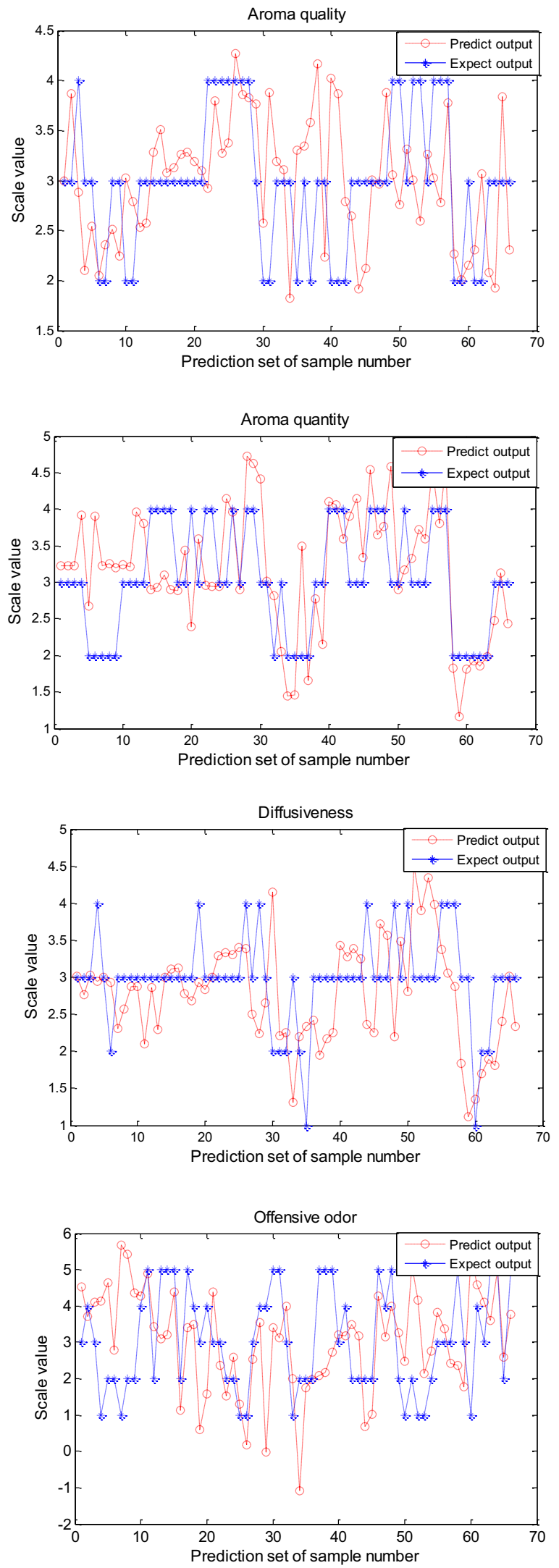

Fig. 4 Comparison diagram for predicted value and expected value of inspection specimens for sensory aroma characteristic indices of flue-cured tobacco leaves 
Table 4 Regression analyses on routine chemical indices and SAC indices of FCTL

\begin{tabular}{|c|c|c|c|c|}
\hline \multirow[t]{2}{*}{ Factors } & \multirow[t]{2}{*}{ Regression equation } & \multicolumn{2}{|c|}{ Significant factor test } & \multirow[t]{2}{*}{ Value $\mathrm{F}$} \\
\hline & & $\begin{array}{l}\text { Partial regres- } \\
\text { sion coefficient }\end{array}$ & $T$ test & \\
\hline \multirow[t]{4}{*}{$\mathrm{Y}_{1}$ /aroma quality } & \multirow[t]{4}{*}{$Y_{1}=-0.35+0.11 X_{1}+0.09 X_{3}+0.15 X_{4}+0.61 X_{5}$} & 0.575 & $10.21^{* *}$ & \multirow[t]{4}{*}{$35.88^{* *}$} \\
\hline & & 0.115 & $1.97^{*}$ & \\
\hline & & 0.165 & $2.43^{*}$ & \\
\hline & & 0.289 & $4.39^{* *}$ & \\
\hline \multirow[t]{3}{*}{$\mathrm{Y}_{2}$ /aroma quantity } & \multirow[t]{3}{*}{$Y_{2}=0.56+0.06 X_{1}+0.47 X_{3}-0.02 X_{9}$} & 0.436 & $7.05^{* *}$ & \multirow[t]{3}{*}{$31.14^{* *}$} \\
\hline & & 0.545 & $9.47^{* *}$ & \\
\hline & & -0.169 & $2.49^{*}$ & \\
\hline \multirow[t]{4}{*}{$\mathrm{Y}_{3} /$ diffusiveness } & \multirow[t]{4}{*}{$Y_{3}=0.50+0.08 X_{1}+0.22 X_{3}+0.19 X_{4}-0.05 X_{9}$} & 0.454 & $7.40^{* *}$ & \multirow[t]{4}{*}{$28.30^{* *}$} \\
\hline & & 0.271 & $4.08^{* *}$ & \\
\hline & & 0.168 & $2.47^{*}$ & \\
\hline & & -0.301 & $4.59^{* *}$ & \\
\hline \multirow[t]{3}{*}{$\mathrm{Y}_{4}$ /offensive odor } & \multirow[t]{3}{*}{$Y_{4}=5.90-0.07 X_{1}-0.08 X_{7}-0.95 X_{8}$} & -0.168 & $2.49^{*}$ & \multirow[t]{3}{*}{$11.77^{* *}$} \\
\hline & & -0.145 & $1.73^{*}$ & \\
\hline & & -0.152 & $1.94^{*}$ & \\
\hline
\end{tabular}

$X_{1}$ is the total sugar; $X_{2}$ is the reducing sugar; $X_{3}$ is the nicotine; $X_{4}$ is the potassium; $X_{5}$ is the chlorine; $X_{6}$ is the ratio of total sugar and reducing sugar; $X_{7}$ is the ratio of sugar and nicotine; $X_{8}$ is the ratio of nitrogen and nicotine; $X_{9}$ is the ratio of potassium and chlorine
Table 5 Comparison analysis on back- propagation neural network model and stepwise regression analysis model

\begin{tabular}{llllll}
\hline Factor & Model & R2 & MSE & RMSE & MAE \\
\hline Aroma quality & BPNN & 0.86 & 0.57 & 0.76 & 0.55 \\
& SRA & 0.40 & 0.69 & 0.83 & 1.23 \\
Aroma quantity & BPNN & 0.81 & 0.53 & 0.73 & 0.54 \\
& SRA & 0.51 & 0.80 & 0.89 & 1.35 \\
Diffusiveness & BPNN & 0.79 & 0.61 & 0.78 & 0.62 \\
& SRA & 0.35 & 0.78 & 0.88 & 1.26 \\
Offensive odor & BPNN & 0.76 & 1.87 & 1.37 & 1.09 \\
& SRA & 0.14 & 4.52 & 2.13 & 3.28 \\
\hline
\end{tabular}

were above 0.7, indicating that the observed values had a good fit with the model. Among these, the aroma quality model was the best, while the offensive odor model was less than ideal. This may be because offensive odor is substantially affected by subjective factors. (3) The model parameters MSE, RMSE and MAE were the important factors in evaluating the predication accuracy; the smaller these parameter values are, the smaller the differences are between the predicted values and the expected values. The MSE, RMSE and MAE indices for the BPNN model of aroma quality improved by $17 \%, 8 \%$ and $55 \%$, respectively compared to those of the SRA model. The aroma quantity index improved by $21 \%, 18 \%$ and $60 \%$, respectively; the diffusiveness index improved by $22 \%, 11 \%$ and $51 \%$, respectively; and the offensive odor index improved by $59 \%, 36 \%$ and $67 \%$, respectively.

\section{Discussion}

In this study, the routine chemical indexes were firstly analyzed and screened out, where total sugar, reducing sugar, nicotine, potassium ion, chloridion, ratio of total sugar and reducing sugar, ratio of sugar and alkali, ratio of nitrogen and alkali, and ratio of potassium and chlorine were significant related with the sensory smell characteristic indexes. It indicated that such 9 routine chemical indexes had significant influence on model output. Since the study adopted the BPNN model, if there were many variables unrelated to model output, an excess model training would be caused; Furthermore, fitting excess data noise in the model and model complicacy may cause the reduction of specimen's predictive ability, and further result in worse generalization ability and robustness. Li et al. utilized principal component analysis (PCA) to make dimensionality reduction for variables and build regression model, which solved some problems to some extent, but may cause use inconvenience and difficulty [13]. Nie et al. adopted the mean impact value (MIV) method to screen out variables, and then built MIV-SVM prediction model, which avoided the curse of dimension ability in a sense, but is very hard to implement for large scale training [18]. In this study, the frequently-used method to screen out variable was adopted to make correlation analysis.

BPNN belongs to supervised learning, and stepwise regression belongs to unsupervised learning and the existing studies do not aim at the comparison of supervised learning and unsupervised learning $[3,21,37]$. In this 
study, a tobacco smell characteristic index model based on nonlinear BPNN was built, to which the input parameters were the routine chemical indexes, and the output parameters were smell mass, smell volume, transmissivity and air impurity. The results showed that the method to predict tobacco smell characteristics by using BPNN model was feasible. However, from a partial perspective, the simulated values of aroma quantity and offensive odor deviated somewhat from the measured values for several possible reasons. First, the aroma quantity may be subdivided into pleasure, fineness, roundness and stretch, while offensive odor can be subdivided into the following smells: withered-scorched, soil texture, earthworm, rosin, pollen, herb and metal and so on. Thus, the output parameter indicators are complex, and the instability in the evaluation has certain effects [14]. Second, the differences may be due to limitations of the BPNN $[34,36]$. In this study, relevant research methods were adopted to screen variables to reduce excessive data noise problems in the network. However, such variable selection can cause problems such as reduced prediction ability, reduced generalizability and poor robustness.

Neural network is established based on the massive training data; its accuracy is usually higher than the nontrained traditional model, especially the utilization of the BPNN model with the optimized hidden layer showed the excellent generalization ability of BP network model [11]. However, in this study, the input parameters of specimen data are only limited to the routine chemical indexes. According to the relevant data, 5868 chemical components had already been identified in tobacco, but the routine chemical indexes were only several [31]. Besides, the output parameters were influenced by subjective and objective factors, and then were not very accurate, causing the model to be built to be "coarse". In future tests, simulation models will be further improved, and the neutral smell index model related to the smell characteristic index will be added to improve result practicability.

\section{Conclusions}

In this study, correlation analyses were first used to analyze and filter the routine chemical indices, and the results are shown as follows: (1) The 9 routine chemical including indicestotal sugar, reducing sugar, nicotine, potassium, chlorine, the ratio of total sugar to reducing sugar, the ratio of sugar to nicotine, the ratio of nitrogen to nicotine, and the ratio of potassium to chlorine were significantly related to the sensory aroma characteristic indices and significantly influenced the model output. (2) The predicting SAC using a BPNN model is not only feasible but its accuracy was significantly higher than that of the traditional
SRA model with the $\mathrm{R} 2$ values of all the indices in the BPNN were above 0.7. (3) Using the optimal regression equations established by the SRA model, a reasonable performance level was achieved, but the SRA was not a suitable model for predicting tobacco aroma characteristics.

Acknowledgement This work was supported by the grant from the National Natural Science Foundation of China (Grant nos. 41601330), Yunnan Science and Technology Innovation Project (Grant nos. 2019HB068), Yunnan Ten Thousand People Program (Congming Zou), Yunnan Applied Basic Research Projects (Grant nos. 2017FB074) and Yunnan Provincial Tobacco Monopoly Bureau China (nos. 2019530000241019).

Authors' contributions Congming Zou conceived the original research plans. Yonglei Jiang and Ke Ren designed the experiments. Binbin $\mathrm{Hu}$ and Chenggang He performed the experiments. Gaokun Zhao and Ruoxing Chen analyzed the data. Yi Chen and Conglian He wrote the manuscript. All authors reviewed and approved the final manuscript.

Funding This study was funded by the grant from the National Natural Science Foundation of China (Grant nos. 41601330), Yunnan Science and Technology Innovation Project (Grant nos. 2019HB068), Yunnan Ten Thousand People Program (Congming Zou), Yunnan Applied Basic Research Projects (Grant nos. 2017FB074) and Yunnan Provincial Tobacco Monopoly Bureau China (nos. 2019530000241019).

Availability of data and materials All data generated or analyzed during this study are included in this published article [and its supplementary information files]

\section{Compliance with ethical standards}

Conflict of interest The authors declare that they have no competing interests. All authors approved the final manuscript.

\section{References}

1. Aghbashlo M, Kianmehr MH, Nazghelichi T, Rafiee S (2011) Optimization of an artificial neural network topology for predicting drying kinetics of carrot cubes using combined response surface and genetic algorithm. Dry Technol 29:770-779. https:// doi.org/10.1080/07373937.2010.538819

2. An J, He G, Qin F, Li R, Huang Z (2018) A new framework of global sensitivity analysis for the chemical kinetic model using PSO-BPNN. Comput Chem Eng 112:154-164. https://doi. org/10.1016/j.compchemeng.2018.02.003

3. Bhattacharjee S, Kishore S, Swetapadma A (2018) A comparative study of supervised learning techniques for human activity monitoring using smart sensors. In: 2018 Second international conference on advances in electronics, computers and communications (ICAECC). IEEE, pp 1-4. https://doi.org/10.1109/icaec c.2018.8479436

4. Biswanath M, Zafar M, Park HS (2013) Characterization of codigestion of industrial sludges for biogas production by artificial neural network and statistical regression models. Environ Technol 34:2145-2153. https://doi.org/10.1080/09593 330.2013 .819022 
5. Cancilla JC, Wang SC, Pablo DR, Gemma M, Cancilla JD, Dan F, Torrecilla JS (2014) Linking chemical parameters to sensory panel results through neural networks to distinguish olive oil quality. J Agric Food Chem 62:10661-10665. https://doi. org/10.1021/jf503482h

6. Dai YH, Han DM, Dai WH (2014) Modeling and computing of stock index forecasting based on neural network and markov chain. Stat World J. https://doi.org/10.1155/2014/124523

7. Du J, Zhang N, Xu ZC, Dai Y, Li DL, Qin L (2011) Relationships between sensory quality and main chemical components in different leaves of flue-cured tobacco. J Zhengzhou Univ Light Ind (Natural Sci). http://en.cnki.com.cn/Article_en/CJFDTOTALZZQB201102005.htm

8. Fang Y, Pang M, Wang B (2017) A course control system of unmanned surface vehicle (USV) using back-propagation neural network (BPNN) and artificial bee colony (ABC) algorithm. Proc Comput Sci 111:361-366. https://doi.org/10.1016/j.procs .2017 .06 .035

9. Gu J, Yin G, Huang P, Guo J, Chen L (2017) An improved back propagation neural network prediction model for subsurface drip irrigation system. Comput Electr Eng 60:58-65. https://doi. org/10.1016/j.compeleceng.2017.02.01

10. Horwitz W, Senzel A, Reynolds H, Park DL (1975) Official methods of analysis of the association of official analytical chemists. VA Association of Official Analytical Chemists, Arlington. https:// doi.org/10.1002/jps.2600600253

11. Ju CY, Cai TJ (2006) Forest volume estimate based on bayesian regularization back propagation neural network. Scientia Silvae Sinicae 12

12. Khatee $A M$, Kasiri MB (2010) Artificial neural networks modeling of contaminated water treatment process by homogeneous and heterogeneous nanocatalysis. J Mol Catal A: Chem 331:86-100. https://doi.org/10.1016/j.molcata.2010.07.016

13. Li Q, Zhou JH, Yang RS, Xia KB, Xie Y, Liu JH, Zhang YY (2011) Evaluation of smoke quality of C3F flue-cured tobacco from Qujing area using model based on principal component regression. Acta Tabacaria Sin 17:26-31

14. Luo DS, Wang B, Qiao XY (2019) National flue-cured tobacco leaf scent style division. Acta Tabacaria Sin. https://doi.org/10.16472 /j.chinatobacco.2019.218

15. Luo Y, Lin B, Wen CB, Luo M (2018) Conducting a correlation model between TCM constitution and physical examination index based on BPNN algorithm. Digit Chin Med 1(1):84-89

16. Madadlou A, Emam DZ, Mousavi ME, Ehsani M, Javanmard M, Sheehan D (2009) Response surface optimization of an artificial neural network for predicting the size of re-assembled casein micelles. Comput Electron Agric 68:216-221. https://doi. org/10.1016/j.compag.2009.06.005

17. Mohammad K, Reza AC (2014) Prediction of some physical and drying properties of terebinth fruit using artificial neural networks. Acta Scientiarum Polonorum Technologia Alimentaria 13:65-78. https://doi.org/10.17306/J.AFS.2014.1.6

18. Nie M, Zhou JH, Yang RS et al (2014) MIV-SVM-based prediction model for smoking quality of flue-cured tobacco. Acta Tabacaria $\operatorname{Sin} 20: 56-62$

19. Pani AK, Mohanta HK (2015) Online monitoring and control of particle size in the grinding process using least square support vector regression and resilient back propagation neural network. ISA Trans 56:206-221. https://doi.org/10.1016/j.isatr a.2014.11.011

20. Peng QR, Cai YQ, Wang DS, Hui JQ, Tang K (2005) Applying BP neural network model to identify leaf tobacco quality based on routine chemical analysis. Acta Tabacaria Sin 11:19-25
21. Perner P, Zscherpel U, Jacobsen C (2001) A comparison between neural networks and decision trees based on data from industrial radiographic testing. Pattern Recogn Lett 22:47-54. https ://doi.org/10.1016/S0167-8655(00)00098-2

22. Seema C, Shrlvatava RK (2009) Performance evaluation of reference evapotranspiration estimation using climate based methods and artificial neural networks. Water Resour Manag 23:825-837. https://doi.org/10.1007/s11269-008-9301-5

23. Sharma MJ, Yu SJ (2015) Stepwise regression data envelopment analysis for variable reduction. Appl Math Comput 253:126-134. https://doi.org/10.1016/j.amc.2014.12.050

24. Song MA, Marian C, Brasky TM, Reisinger S, Djordjevic M, Shields PG (2016) Chemical and toxicological characteristics of conventional and low-TSNA moist snuff tobacco products. Toxicol Lett 245:68-77. https://doi.org/10.1016/j.toxlet.2016.01.012

25. Sugiono S, Soenoko R, Lukodono RP (2018) Static/dynamic zoometry concept to design cattle facilities using back propagation neural network (BPNN). Artif Intell Emerg Trends Appl. https://doi.org/10.5722/intechopen.75136

26. Sun JG, He JW, Wu FG, Tu SX, Yan TJ, Hui SI, Hao XIE (2011) Comparative analysis on chemical components and sensory quality of aging flue-cured tobacco from four main tobacco areas of China. Agric Sci China 10(8):1222-1231. https://doi.org/10.1016/ S1671-2927(11)60113-2

27. Sun SP, Yi DQ, Jiang Y, Wu CP, Zang B, Li Y (2011) Prediction of formation enthalpies for Al2X-type intermetallics using backpropagation neural network. Mater Chem Phys 126:632-641. https://doi.org/10.1016/j.matchemphys.2011.01.004

28. Tong Z, Xiao B, Jiao F, Fang D, Zeng J, Wu X, Li Y et al (2016) Large-scale development of SSR markers in tobacco and construction of a linkage map in flue-cured tobacco. Breed Sci. https://doi.org/10.1270/jsbbs.15129

29. Torrecilla JS, Cancilia JC, Matute G, Diaz-rodriguez P (2013) Neural network models to classify olive oils within the protected denomination of origin framework. Int J Food Sci Technol 48:2528-2534. https://doi.org/10.1111/ijfs.12245

30. Tso G, Yau K (2007) Predicting electricity energy consumption: a comparison of regression analysis, decision tree and neural networks. Energy 32:1761-1768. https://doi.org/10.1016/j.energ y.2006.11.010

31. Yan KY (2002) Tobacco chemistry. Zhengzhou University Press, Henan

32. Ye XF, Liu HE, Ye XW, Zhang T, Zhang RN, Qu JK, Yu JJ (2013) Distribution characteristics of osmiophilic granules' content in tobacco leaves at different altitudes and the relationship with the content of neutral aroma components. J Food Agric Environ 11:1176-1181

33. Yu H, Zheng R, Wang Z, Bu W (2015) Application of neural network based PID method for temperature control of aquaculture greenhouse. In: 2015 ASABE annual international meeting. American Society of Agric. and Biol. Eng., p 1. https://doi. org/10.13031/aea.32.11444

34. Zhang C, Liu F, Kong W, Cui P, He Y, Zhou W (2016) Estimation and visualization of soluble sugar content in oilseed rape leaves using hyperspectral imaging. Trans ASABE 59(6):1499-1505. https://doi.org/10.13031/trans.59.10485

35. Zhang F, Zhang X (2011) Classification and quality evaluation of tobacco leaves based on image processing and fuzzy comprehensive evaluation. Sensors 11(3):2369-2384. https://doi. org/10.3390/s110302369

36. Zhang R, Wang YW, Zhang MZ (2019) Automatic test oracle based on probabilistic neural networks. In: Recent developments in intelligent computing, communication 
and devices. Springer, Singapore, pp 437-445. https://doi. org/10.1007/978-981-10-8944-2_50

37. Zhao PF, Liu C, Feng $X$ et al (2019) Stochastic seismic inversion based on neural network. Chin J Geophys 62:1172-1180
Publisher's Note Springer Nature remains neutral with regard to jurisdictional claims in published maps and institutional affiliations. 